\title{
New mean value theorems and generalization of Hadamard inequality via coordinated $m$-convex functions
}

\author{
G Farid $^{1}$, M Marwan$^{2}$ and Atiq Ur Rehman ${ }^{1 *}$
}

"Correspondence:
atiq@mathcity.org
${ }^{1}$ COMSATS Institute of Information
Technology, Kamra Road, Attock,
43600, Pakistan
Full list of author information is
available at the end of the article

available at the end of the article

\begin{abstract}
We derive new mean value theorems for functionals associated with Hadamard inequality for convex functions on the coordinates. We present some Hadamard type inequalities and related results for $m$-convex functions on the coordinates.
\end{abstract}

Keywords: convex functions; $m$-convex functions; convex functions on coordinates; Hadamard inequality; mean value theorems

\section{Introduction}

We start with the very basic concept of a convex function that has been seen as important ever since it was defined.

Definition 1.1 A real valued function $f: I \rightarrow \mathbb{R}$, where $I$ is an interval in $\mathbb{R}$, is called convex if

$$
f(\lambda x+(1-\lambda) y) \leq \lambda f(x)+(1-\lambda) f(y)
$$

where $\lambda \in[0,1]$, and $x, y \in I$.

One cannot deny the importance of convex functions. It is used by many mathematicians in many fields of mathematics such as functional analysis, mathematical statistics, and complex analysis (see for example [1-4] and references therein). In the field of inequalities, convex functions play a unique role. Most of the new inequalities till now defined are results and consequences of (1). The most cardinal and classical inequality for convex functions is stated in the following.

Theorem 1.2 Let $f: I \rightarrow \mathbb{R}$ be convex function and $a, b \in I$ with $a<b$, then

$$
f\left(\frac{a+b}{2}\right) \leq \frac{1}{b-a} \int_{a}^{b} f(x) d x \leq \frac{f(a)+f(b)}{2}
$$

This famous integral inequality can be traced back to the papers presented by Hermite [5] and Hadamard [6]. Researchers have used inequality (2) several times for giving generalization and modification of Hadamard type inequalities using different modification

(C) 2015 Farid et al. This article is distributed under the terms of the Creative Commons Attribution 4.0 International License (http://creativecommons.org/licenses/by/4.0/), which permits unrestricted use, distribution, and reproduction in any medium, provided you give appropriate credit to the original author(s) and the source, provide a link to the Creative Commons license, and indicate if changes were made. 
of convex functions. For refinements, counterparts, and generalizations see for example $[3,7-19]$.

Recently, many authors have considered a convex function on the coordinates to give the Hadamard inequality on the coordinates and its different variants. Also they have given many results associated with it (e.g. see [20-23]).

Definition 1.3 Let $\Delta^{2}:=[a, b] \times[c, d] \subset \mathbb{R}^{2}$ with $a<b$ and $c<d$. A function $f: \Delta^{2} \rightarrow \mathbb{R}$ is called convex on the coordinates if the partial mapping $f_{y}:[a, b] \rightarrow \mathbb{R}, f_{y}(u):=f(u, y)$ and $f_{x}:[c, d] \rightarrow \mathbb{R}, f_{x}(v):=f(x, v)$ are convex, where they are defined for all $y \in[c, d]$ and $x \in[a, b]$.

We will keep the notation $\Delta^{2}=[a, b] \times[c, d]$ throughout this paper.

Recall that a mapping $f: \Delta^{2} \rightarrow \mathbb{R}$ is convex in $\Delta^{2}$ if, for $(x, y),(u, v) \in \Delta^{2}$ and $\alpha \in[0,1]$, the following inequality holds:

$$
f(\alpha(x, y)+(1-\alpha)(u, v)) \leq \alpha f(x, y)+(1-\alpha) f(u, v) .
$$

It can be seen that every convex mapping $f: \Delta^{2} \rightarrow \mathbb{R}$ is convex on the coordinates but the converse is not true. Dragomir gave the Hadamard inequality for a rectangle in the plane for convex functions on the coordinates (see [11]).

Theorem 1.4 Suppose that $f: \Delta^{2} \rightarrow \mathbb{R}$ is convex on the coordinates on $\Delta^{2}$. Then one has the following inequalities:

$$
\begin{aligned}
f( & \left.\frac{a+b}{2}, \frac{c+d}{2}\right) \\
\leq & \frac{1}{2}\left[\frac{1}{b-a} \int_{a}^{b} f\left(x, \frac{c+d}{2}\right) d x+\frac{1}{d-c} \int_{c}^{d} f\left(\frac{a+b}{2}, y\right) d y\right] \\
\leq & \frac{1}{(b-a)(d-c)} \int_{a}^{b} \int_{c}^{d} f(x, y) d x d y \\
\leq & \frac{1}{4}\left[\frac{1}{b-a} \int_{a}^{b} f(x, c) d x+\frac{1}{b-a} \int_{a}^{b} f(x, d) d x+\frac{1}{d-c} \int_{c}^{d} f(a, y) d y\right. \\
& \left.+\frac{1}{d-c} \int_{c}^{d} f(b, y) d y\right] \\
\leq & \frac{1}{4}[f(a, c)+f(a, d)+f(b, c)+f(b, d)] .
\end{aligned}
$$

In [24], Toader defined the concept of $m$-convexity, an intermediate between the usual convexity and star shape properties.

Definition 1.5 The function $f:[0, b] \rightarrow \mathbb{R}$ is said to be $m$-convex, where $m \in[0,1]$, if for every $x, y \in[0, b]$ and $t \in[0,1]$ we have

$$
f(t x+m(1-t) y) \leq t f(x)+m(1-t) f(y) .
$$

In [25] using inequality (2) and (3) Dragomir and Toader gave the following Hadamard type inequality for $m$-convex functions. 
Theorem 1.6 Let $f:[0, \infty) \rightarrow \mathbb{R}$ be m-convex function with $m \in(0,1]$ and $0 \leq a<b$. If $f \in L_{1}[a, b]$, then one has the following inequalities:

$$
\begin{aligned}
f\left(\frac{a+b}{2}\right) & \leq \frac{1}{b-a} \int_{a}^{b} \frac{f(x)+m f\left(\frac{x}{m}\right)}{2} d x \\
& \leq \frac{m+1}{4}\left[\frac{f(a)+f(b)}{2}+m\left(\frac{f\left(\frac{a}{m}\right)+f\left(\frac{b}{m}\right)}{2}\right)\right]
\end{aligned}
$$

They also gave the following related results to the Hadamard type inequality for $m$ convex functions.

Theorem 1.7 Let $f:[0, \infty) \rightarrow \mathbb{R}$ be an m-convex function with $m \in(0,1]$. If $0 \leq a<b<\infty$ and $f \in L_{1}[a, b]$, then one has the inequality

$$
\frac{1}{b-a} \int_{a}^{b} f(x) d x \leq \min \left\{\frac{f(a)+m f\left(\frac{b}{m}\right)}{2}, \frac{f(b)+m f\left(\frac{a}{m}\right)}{2}\right\} .
$$

Theorem 1.8 Letf $:[0, \infty) \rightarrow \mathbb{R}$ be an m-convex function with $m \in(0,1]$. If $0 \leq a<b<\infty$ and $f$ is differentiable on $(0, \infty)$, then one has the inequality

$$
\begin{aligned}
\frac{f(m b)}{m}-\frac{b-a}{2} f^{\prime}(m b) & \leq \frac{1}{b-a} \int_{a}^{b} f(x) d x \\
& \leq \frac{(b-m a) f(d)-(a-m b) f(a)}{2(b-a)} .
\end{aligned}
$$

In this paper, new mean value theorems of Cauchy type for functionals associated with nonnegative differences of the Hadamard inequality on the coordinates are proved. Generalized results related to the Hadamard inequality for $m$-convex functions on the coordinates are also given.

\section{Mean value theorems}

We know that if a function $f$ is twice differentiable on an interval $I$ then it is convex on $I$ if and only if its second order derivative is nonnegative. If a function $f(X):=f(x, y)$ has continuous second order partial derivatives on interior of $\Delta^{2}$ then it is convex on $\Delta^{2}$ if the Hessian matrix

$$
H_{f}(X)=\left(\begin{array}{ll}
\frac{\partial^{2} f(X)}{\partial x^{2}} & \frac{\partial^{2} f(X)}{\partial y \partial x} \\
\frac{\partial^{2} f(X)}{\partial x \partial y} & \frac{\partial^{2} f(X)}{\partial y^{2}}
\end{array}\right)
$$

is nonnegative definite, that is, $\mathbf{v} H_{f}(X) \mathbf{v}^{\tau}$ is nonnegative for all real nonnegative vector $\mathbf{v}$ (see [3], p.11).

It is easy to see that $f: \Delta^{2} \rightarrow \mathbb{R}$ is coordinated convex on $\Delta^{2}$ iff

$$
f_{x}^{\prime \prime}(y)=\frac{\partial^{2} f(x, y)}{\partial y^{2}} \quad \text { and } \quad f_{y}^{\prime \prime}(x)=\frac{\partial^{2} f(x, y)}{\partial x^{2}}
$$

are nonnegative for all interior points $(x, y)$ in $\Delta^{2}$. 
For a real valued function $f: \Delta^{2} \rightarrow \mathbb{R}$ we define

$$
\begin{aligned}
\mathcal{H}(f)= & \frac{1}{2}\left[\frac{1}{b-a} \int_{a}^{b} f\left(x, \frac{c+d}{2}\right) d x+\frac{1}{d-c} \int_{c}^{d} f\left(\frac{a+b}{2}, y\right) d y\right] \\
& -f\left(\frac{a+b}{2}, \frac{c+d}{2}\right) .
\end{aligned}
$$

One can note that $\mathcal{H}(f) \geq 0$ if $f$ is convex on the coordinates in $\Delta^{2}$.

To give the mean value theorems of Cauchy type, we need the following lemma.

Lemma 2.1 Let $f: \Delta^{2} \rightarrow \mathbb{R}$ be a function such that

$$
m_{1} \leq \frac{\partial^{2} f(x, y)}{\partial x^{2}} \leq M_{1} \quad \text { and } \quad m_{2} \leq \frac{\partial^{2} f(x, y)}{\partial y^{2}} \leq M_{2}
$$

for all interior points $(x, y)$ in $\Delta^{2}$.

Consider the functions $g, h: \Delta^{2} \rightarrow \mathbb{R}$ defined as

$$
g(x, y)=\frac{1}{2} \max \left\{M_{1}, M_{2}\right\}\left(x^{2}+y^{2}\right)-f(x, y)
$$

and

$$
h(x, y)=f(x, y)-\frac{1}{2} \min \left\{m_{1}, m_{2}\right\}\left(x^{2}+y^{2}\right) .
$$

Then $g$ and $h$ are coordinated convex in $\Delta^{2}$.

Proof Since

$$
\frac{\partial^{2} g(x, y)}{\partial x^{2}}=\max \left\{M_{1}, M_{2}\right\}-\frac{\partial^{2} f(x, y)}{\partial x^{2}} \geq 0
$$

and

$$
\frac{\partial^{2} g(x, y)}{\partial y^{2}}=\frac{\partial^{2} f(x, y)}{\partial y^{2}}-\min \left\{m_{1}, m_{2}\right\} \geq 0
$$

for all interior points $(x, y)$ in $\Delta^{2}, g$ is convex on the coordinates in $\Delta^{2}$.

Similarly one can prove that $h$ is convex on the coordinates in $\Delta^{2}$.

Theorem 2.2 Let $f: \Delta^{2} \rightarrow \mathbb{R}$ be a function, which has continuous partial derivatives of second order in $\Delta^{2}$ and $q(x, y):=x^{2}+y^{2}$. Then there exist $\left(\eta_{1}, \xi_{1}\right)$ and $\left(\eta_{2}, \xi_{2}\right)$ in the interior of $\Delta^{2}$ such that

$$
\mathcal{H}(f)=\frac{1}{2} \frac{\partial^{2} f\left(\eta_{1}, \xi_{1}\right)}{\partial x^{2}} \mathcal{H}(q)
$$

and

$$
\mathcal{H}(f)=\frac{1}{2} \frac{\partial^{2} f\left(\eta_{2}, \xi_{2}\right)}{\partial x^{2}} \mathcal{H}(q)
$$

provided that $\mathcal{H}(q) \neq 0$. 
Proof Since $f$ has continuous partial derivatives of second order in compact set $\Delta^{2}$, there exist real numbers $m_{1}, m_{2}, M_{1}$, and $M_{2}$ such that

$$
m_{1} \leq \frac{\partial^{2} f(x, y)}{\partial x^{2}} \leq M_{1} \quad \text { and } \quad m_{2} \leq \frac{\partial^{2} f(x, y)}{\partial y^{2}} \leq M_{2} .
$$

Now consider functions $g$ defined in Lemma 2.1. As $g$ is convex on the coordinates in $\Delta^{2}$,

$$
\mathcal{H}(g) \geq 0
$$

that is,

$$
\mathcal{H}\left(\frac{1}{2} \max \left\{M_{1}, M_{2}\right\} q-f(x, y)\right) \geq 0 .
$$

From this we get

$$
2 \mathcal{H}(f) \leq \max \left\{M_{1}, M_{2}\right\} \mathcal{H}(q) .
$$

On the other hand, for the function $h$, one has

$$
\min \left\{m_{1}, m_{2}\right\} \mathcal{H}(q) \leq 2 \mathcal{H}(f)
$$

As $\mathcal{H}(q) \neq 0$, combining inequalities (6) and (7), we get

$$
\min \left\{m_{1}, m_{2}\right\} \leq \frac{2 \mathcal{H}(f)}{H(q)} \leq \max \left\{M_{1}, M_{2}\right\}
$$

Then there exist $\left(\eta_{1}, \xi_{1}\right)$ and $\left(\eta_{2}, \xi_{2}\right)$ in the interior of $\Delta^{2}$ such that

$$
\frac{2 \mathcal{H}(f)}{H(q)}=\frac{\partial^{2} f\left(\eta_{1}, \xi_{1}\right)}{\partial x^{2}} \quad \text { and } \quad \frac{2 \mathcal{H}(f)}{H(q)}=\frac{\partial^{2} f\left(\eta_{2}, \xi_{2}\right)}{\partial y^{2}}
$$

Hence the required result follows.

\section{Hadamard type inequalities for $\boldsymbol{m}$-convex function on two coordinates}

In this section we give Hadamard type inequalities for $m$-convex functions on two coordinates. First of all we give the definitions of $m$-convex functions in two coordinates.

Definition 3.1 Let $\Delta=[0, b] \times[0, d] \subset[0, \infty)^{2}$, then a function $f: \Delta \rightarrow \mathbb{R}$, will be called $m$-convex on the coordinates if the partial mappings $f_{y}:[0, b] \rightarrow \mathbb{R}, f_{y}(u):=f(u, y)$, and $f_{x}:[0, d] \rightarrow \mathbb{R}, f_{x}(v):=f(x, v)$, are $m$-convex on $[0, b]$ and $[0, d]$, respectively.

In the following we give the Hadamard type inequality for $m$-convex functions on the coordinates.

Theorem 3.2 Let $\Delta=[0, b] \times[0, d] \subset[0, \infty)^{2}$ with $b, d>0$ and $f: \Delta \rightarrow \mathbb{R}$ be m-convex on the coordinates in $\Delta$ with $m \in(0,1]$. If $f_{x} \in L_{1}[0, d]$ and $f_{y} \in L_{1}[0, b], 0 \leq a<b, 0 \leq c<d$. 
Then we have

$$
\begin{aligned}
\frac{1}{b-a} & \int_{a}^{b} f\left(x, \frac{c+d}{2}\right) d x+\frac{1}{d-c} \int_{c}^{d} f\left(\frac{a+b}{2}, y\right) d y \\
\leq & \frac{1}{2(b-a)(d-c)} \int_{a}^{b} \int_{c}^{d}\left(2 f(x, y)+m\left(f\left(x, \frac{y}{m}\right)+f\left(\frac{x}{m}, y\right)\right)\right) d y d x \\
\leq & \frac{(m+1)^{2}}{16}\left[f(a, c)+f(b, c)+f(a, d)+f(b, d)+m\left(f\left(\frac{a}{m}, c\right)+f\left(\frac{b}{m}, c\right)\right.\right. \\
& \left.+f\left(\frac{a}{m}, d\right)+f\left(\frac{b}{m}, d\right)+f\left(a, \frac{c}{m}\right)+f\left(a, \frac{d}{m}\right)+f\left(b, \frac{c}{m}\right)+f\left(b, \frac{d}{m}\right)\right) \\
& \left.+m^{2}\left(f\left(\frac{a}{m}, \frac{c}{m}\right)+f\left(\frac{b}{m}, \frac{c}{m}\right)+f\left(\frac{a}{m}, \frac{d}{m}\right)+f\left(\frac{b}{m}, \frac{d}{m}\right)\right)\right] .
\end{aligned}
$$

Proof Since mapping $f: \Delta \rightarrow \mathbb{R}$ is $m$-convex on the coordinates, the functions $f_{x}$ and $f_{y}$ are $m$-convex on $[0, d]$ and $[0, b]$, respectively. Using (4) for the function $f_{y}$ we have

$$
\begin{aligned}
f_{y}\left(\frac{a+b}{2}\right) & \leq \frac{1}{b-a} \int_{a}^{b}\left(\frac{f_{y}(x)+m f_{y}\left(\frac{x}{m}\right)}{2}\right) d x \\
& \leq \frac{m+1}{4}\left[\frac{f_{y}(a)+f_{y}(b)}{2}+m \frac{f_{y}\left(\frac{a}{m}\right)+f_{y}\left(\frac{b}{m}\right)}{2}\right]
\end{aligned}
$$

that is,

$$
\begin{aligned}
f\left(\frac{a+b}{2}, y\right) & \leq \frac{1}{b-a} \int_{a}^{b}\left(\frac{f(x, y)+m f\left(\frac{x}{m}, y\right)}{2}\right) d x \\
& \leq \frac{m+1}{4}\left[\frac{f(a, y)+f(b, y)}{2}+m \frac{f\left(\frac{a}{m}, y\right)+f\left(\frac{b}{m}, y\right)}{2}\right] .
\end{aligned}
$$

From this one has

$$
\begin{aligned}
& \frac{1}{d-c} \int_{c}^{d} f\left(\frac{a+b}{2}, y\right) d y \\
& \quad \leq \frac{1}{(b-a)(d-c)} \int_{a}^{b} \int_{c}^{d}\left(\frac{f(x, y)+m f\left(\frac{x}{m}, y\right)}{2}\right) d y d x \\
& \quad \leq \frac{m+1}{4(d-c)} \int_{c}^{d}\left[\frac{f(a, y)+f(b, y)}{2}+m \frac{f\left(\frac{a}{m}, y\right)+f\left(\frac{b}{m}, y\right)}{2}\right] d y .
\end{aligned}
$$

Similarly using (4) for the function $f_{x}$ we have

$$
\begin{aligned}
& \frac{1}{b-a} \int_{a}^{b} f\left(x, \frac{c+d}{2}\right) d x \\
& \quad \leq \frac{1}{(b-a)(d-c)} \int_{a}^{b} \int_{c}^{d}\left(\frac{f(x, y)+m f\left(x, \frac{y}{m}\right)}{2}\right) d y d x \\
& \leq \frac{m+1}{4(b-a)} \int_{a}^{b}\left[\frac{f(x, c)+f(x, d)}{2}+m \frac{f\left(x, \frac{c}{m}\right)+f\left(x, \frac{d}{m}\right)}{2}\right] d x .
\end{aligned}
$$


By adding (9) and (10), we get

$$
\begin{aligned}
& \frac{1}{b-a} \int_{a}^{b} f\left(x, \frac{c+d}{2}\right) d x+\frac{1}{d-c} \int_{c}^{d} f\left(\frac{a+b}{2}, y\right) d y \\
& \leq \frac{1}{2(b-a)(d-c)} \int_{a}^{b} \int_{c}^{d}\left(2 f(x, y)+m\left(f\left(x, \frac{y}{m}\right)+f\left(\frac{x}{m}, y\right)\right)\right) d y d x \\
& \leq \frac{m+1}{4}\left[\frac{1}{b-a} \int_{a}^{b}\left(\frac{f(x, c)+f(x, d)}{2}+m \frac{f\left(x, \frac{c}{m}\right)+f\left(x, \frac{d}{m}\right)}{2}\right) d x\right. \\
& \left.\quad+\frac{1}{d-c} \int_{c}^{d}\left(\frac{f(a, y)+f(b, y)}{2}+m \frac{f\left(\frac{a}{m}, y\right)+f\left(\frac{b}{m}, y\right)}{2}\right) d y\right]
\end{aligned}
$$

For fixed $y$ using the $m$-convexity of $f_{y}$ we have

$$
f(x, y) \leq \frac{f(x, y)+m f\left(\frac{x}{m}, y\right)}{2}
$$

Performing the average integral over the interval $[a, b]$ and using (4) we get

$$
\begin{aligned}
\frac{1}{b-a} \int_{a}^{b} f(x, y) d x & \leq \frac{1}{b-a} \int_{a}^{b}\left(\frac{f(x, y)+m f\left(\frac{x}{m}, y\right)}{2}\right) d x \\
& \leq \frac{m+1}{4}\left[\frac{f(a, y)+f(b, y)}{2}+m \frac{f\left(\frac{a}{m}, y\right)+f\left(\frac{b}{m}, y\right)}{2}\right]
\end{aligned}
$$

Similarly for fixed $x$ using the $m$-convexity of $f_{x}$ one has

$$
\begin{aligned}
\frac{1}{d-c} \int_{c}^{d} f(x, y) d y & \leq \frac{1}{d-c} \int_{c}^{d}\left(\frac{f(x, y)+m f\left(x, \frac{y}{m}\right)}{2}\right) d y \\
& \leq \frac{m+1}{4}\left[\frac{f(x, c)+f(x, d)}{2}+m \frac{f\left(x, \frac{c}{m}\right)+f\left(x, \frac{d}{m}\right)}{2}\right]
\end{aligned}
$$

Considering (13) for $y=c, d$, (14) for $x=a, b$, then (13) for $y=\frac{c}{m}, \frac{d}{m}$, (14) for $x=\frac{a}{m}, \frac{b}{m}$ to multiply later with $m$. Adding all these inequalities, we obtain

$$
\begin{aligned}
\frac{1}{b-a} & \int_{a}^{b}\left(\frac{f(x, c)+f(x, d)}{2}+m \frac{f\left(x, \frac{c}{m}\right)+f\left(x, \frac{d}{m}\right)}{2}\right) d x \\
& +\frac{1}{d-c} \int_{c}^{d}\left(\frac{f(a, y)+f(b, y)}{2}+m \frac{f\left(\frac{a}{m}, y\right)+f\left(\frac{b}{m}, y\right)}{2}\right) d y \\
\leq & \frac{1}{2}\left[\frac { 1 } { b - a } \int _ { a } ^ { b } \left(\frac{f(x, c)+m f\left(\frac{x}{m}, c\right)+f(x, d)+m f\left(\frac{x}{m}, d\right)}{2}\right.\right. \\
& \left.+m \frac{f\left(x, \frac{c}{m}\right)+m f\left(\frac{x}{m}, \frac{c}{m}\right)+f\left(x, \frac{d}{m}\right)+m f\left(\frac{x}{m}, \frac{d}{m}\right)}{2}\right) d x \\
& +\frac{1}{d-c} \int_{c}^{d}\left(\frac{f(a, y)+m f\left(a, \frac{y}{m}\right)+f(b, y)+m f\left(b, \frac{y}{m}\right)}{2}\right. \\
& \left.\left.+m \frac{f\left(\frac{a}{m}, y\right)+m f\left(\frac{a}{m}, \frac{y}{m}\right)+f\left(\frac{b}{m}, y\right)+m f\left(\frac{b}{m}, \frac{y}{m}\right)}{2}\right) d y\right]
\end{aligned}
$$




$$
\begin{aligned}
\leq & \frac{1}{4}\left[f(a, c)+f(b, c)+f(a, d)+f(b, d)+m\left(f\left(\frac{a}{m}, c\right)+f\left(\frac{b}{m}, c\right)\right.\right. \\
& \left.+f\left(\frac{a}{m}, d\right)+f\left(\frac{b}{m}, d\right)+f\left(a, \frac{c}{m}\right)+f\left(a, \frac{d}{m}\right)+f\left(b, \frac{c}{m}\right)+f\left(b, \frac{d}{m}\right)\right) \\
& \left.+m^{2}\left(f\left(\frac{a}{m}, \frac{c}{m}\right)+f\left(\frac{b}{m}, \frac{c}{m}\right)+f\left(\frac{a}{m}, \frac{d}{m}\right)+f\left(\frac{b}{m}, \frac{d}{m}\right)\right)\right] .
\end{aligned}
$$

Now combining inequalities in (11) and (15), we get the last two inequalities of the theorem.

Theorem 3.3 Let $\Delta=[0, b] \times[0, d] \subset[0, \infty)^{2}$ with $b, d>0$ and $f: \Delta \rightarrow \mathbb{R}$ be m-convex on the coordinate in $\Delta$ with $m \in(0,1]$. If $f_{x} \in L_{1}[0, d]$ and $f_{y} \in L_{1}[0, b]$, then

$$
\begin{aligned}
2 f\left(\frac{a+b}{2}, \frac{c+d}{2}\right) \leq & \frac{1}{b-a} \int_{a}^{b}\left(\frac{f\left(x, \frac{c+d}{2}\right)+m f\left(\frac{x}{m}, \frac{c+d}{2}\right)}{2}\right) d x \\
& +\frac{1}{c-d} \int_{c}^{d}\left(\frac{f\left(\frac{a+b}{2}, y\right)+m f\left(\frac{a+b}{2}, \frac{y}{m}\right)}{2}\right) d y
\end{aligned}
$$

Proof By using (8) for $y=\frac{c+d}{2}$

$$
f\left(\frac{a+b}{2}, \frac{c+d}{2}\right) \leq \frac{1}{b-a} \int_{a}^{b}\left(\frac{f\left(x, \frac{c+d}{2}\right)+m f\left(\frac{x}{m}, \frac{c+d}{2}\right)}{2}\right) d x .
$$

Applying the first inequality in (4) for $f_{x}$ on $[c, d]$ we have

$$
f\left(x, \frac{c+d}{2}\right) \leq \frac{1}{c-d} \int_{c}^{d}\left(\frac{f(x, y)+m f\left(x, \frac{y}{m}\right)}{2}\right) d y .
$$

Put $x=\frac{a+b}{2}$ we get

$$
f\left(\frac{a+b}{2}, \frac{c+d}{2}\right) \leq \frac{1}{c-d} \int_{c}^{d}\left(\frac{f\left(\frac{a+b}{2}, y\right)+m f\left(\frac{a+b}{2}, \frac{y}{m}\right)}{2}\right) d y .
$$

By adding (17) and (18) we get (16).

Remark 3.4 By putting $m=1$ in Theorem 3.2 and Theorem 3.3 and combining we get inequalities in Theorem 1.4.

Theorem 3.5 Let $f, f_{x}$, and $f_{y}$ be defined as in Theorem 3.2. Then one has the following inequality:

$$
\begin{gathered}
\frac{2}{(b-a)(d-c)} \int_{a}^{b} \int_{c}^{d} f(x, y) d y d x \\
\leq \min \left\{\frac{1}{b-a} \int_{a}^{b}\left(\frac{f(x, c)+m f\left(x, \frac{d}{m}\right)}{2}\right) d x,\right. \\
\left.\frac{1}{b-a} \int_{a}^{b}\left(\frac{f(x, d)+m f\left(x, \frac{c}{m}\right)}{2}\right) d x\right\}
\end{gathered}
$$




$$
\begin{aligned}
& +\min \left\{\frac{1}{d-c} \int_{c}^{d}\left(\frac{f(a, y)+m f\left(\frac{b}{m}, y\right)}{2}\right) d y,\right. \\
& \left.\frac{1}{d-c} \int_{c}^{d}\left(\frac{f(b, y)+m f\left(\frac{a}{m}, y\right)}{2}\right) d y\right\} .
\end{aligned}
$$

Proof Since mapping $f: \Delta \rightarrow \mathbb{R}$ is $m$-convex on the coordinates, the functions $f_{x}$ and $f_{y}$ are $m$-convex on $[0, d]$ and $[0, b]$, respectively. Thus we have by Theorem 1.7

$$
\frac{1}{b-a} \int_{a}^{b} f_{y}(x) d x \leq \min \left\{\frac{f_{y}(a)+f_{y}\left(\frac{b}{m}\right)}{2}, \frac{f_{y}(b)+f_{y}\left(\frac{a}{m}\right)}{2}\right\}
$$

that is,

$$
\frac{1}{b-a} \int_{a}^{b} f(x, y) d x \leq \min \left\{\frac{f(a, y)+f\left(\frac{b}{m}, y\right)}{2}, \frac{f(b, y)+f\left(\frac{a}{m}, y\right)}{2}\right\} .
$$

Performing the average integral over the interval $[c, d]$

$$
\begin{aligned}
& \frac{1}{(b-a)(d-c)} \int_{a}^{b} \int_{c}^{d} f(x, y) d x \\
& \leq \min \left\{\frac{1}{d-c} \int_{c}^{d}\left(\frac{f(a, y)+f\left(\frac{b}{m}, y\right)}{2}\right) d y, \frac{1}{d-c} \int_{c}^{d}\left(\frac{f(b, y)+f\left(\frac{a}{m}, y\right)}{2}\right) d y\right\} .
\end{aligned}
$$

Similarly for $f_{x}$ one has

$$
\begin{aligned}
& \frac{1}{(b-a)(d-c)} \int_{a}^{b} \int_{c}^{d} f(x, y) d x \\
& \quad \leq \min \left\{\frac{1}{b-a} \int_{a}^{b}\left(\frac{f(x, c)+f\left(x, \frac{d}{m}\right)}{2}\right) d x, \frac{1}{b-a} \int_{a}^{b}\left(\frac{f(x, d)+f\left(x, \frac{c}{m}\right)}{2}\right) d x\right\} .
\end{aligned}
$$

For the desired result we add inequalities (20) and (21).

Theorem 3.6 Let $f_{x}:[0, d] \subset[0, \infty) \rightarrow \mathbb{R}, f_{x}(u)=f(x, u)$ and $f_{y}:[0, b] \subset[0, \infty) \rightarrow \mathbb{R}$, $f_{y}(v)=f(v, y)$ be partial m-convex mappings with $m \in(0,1]$. If $0 \leq a<b<\infty, 0 \leq c<d<\infty$, also if $f_{x} \in L_{1}[0, d]$ and $f_{y} \in L_{1}[0, b]$ with $f_{y}$ and $f_{x}$ are differentiable on $(0, \infty)$, then we have the following inequality:

$$
\begin{aligned}
\frac{1}{2 m}( & \left.\frac{1}{b-a} \int_{a}^{b} f(x, m d) d x+\frac{1}{d-c} \int_{c}^{d} f(m b, y) d y\right) \\
& -\frac{1}{2}\left(\frac{d-c}{b-a} \int_{a}^{b} \frac{\partial f(x, m d)}{\partial y} d x+\frac{b-a}{d-c} \int_{c}^{d} \frac{\partial f(m b, y)}{\partial x} d y\right) \\
\leq & \frac{1}{(b-a)(d-c)} \int_{a}^{b} \int_{c}^{d} f(x, y) d y d x \leq \frac{1}{4(b-a)(d-c)} \\
& \times\left[(d-m c) \int_{a}^{b} f(x, d) d x+(b-m a) \int_{c}^{d} f(b, y) d y\right. \\
& \left.-\left((c-m d) \int_{a}^{b} f(x, c) d x+(a-m b) \int_{c}^{d} f(a, y) d y\right)\right] .
\end{aligned}
$$


Proof Since the functions $f_{x}$ and $f_{y}$ are $m$-convex on $[0, d]$ and $[0, b]$, respectively, and the functions $f_{x}$ and $f_{y}$ are differentiable on $(0, \infty)$, applying Theorem 1.8 we have

$$
\begin{aligned}
\frac{f_{x}(m d)}{m}-\frac{d-c}{2} f_{x}^{\prime}(m d) & \leq \frac{1}{d-c} \int_{c}^{d} f_{x}(y) d y \\
& \leq \frac{(d-m c) f_{x}(d)-(c-m d) f_{x}(c)}{2(d-c)}
\end{aligned}
$$

and

$$
\begin{aligned}
\frac{f_{y}(m b)}{m}-\frac{b-a}{2} f_{y}^{\prime}(m b) & \leq \frac{1}{b-a} \int_{a}^{b} f_{y}(x) d y \\
& \leq \frac{(b-m a) f_{y}(b)-(a-m b) f_{y}(a)}{2(b-a)} .
\end{aligned}
$$

This gives us

$$
\begin{aligned}
\frac{f(x, m d)}{m}-\frac{d-c}{2} \frac{\partial f(x, m d)}{\partial y} & \leq \frac{1}{d-c} \int_{c}^{d} f(x, y) d y \\
& \leq \frac{(d-m c) f(x, d)-(c-m d) f(x, c)}{2(d-c)}
\end{aligned}
$$

and

$$
\begin{aligned}
\frac{f(m b, y)}{m}-\frac{b-a}{2} \frac{\partial f(m b, y)}{\partial x} & \leq \frac{1}{b-a} \int_{a}^{b} f(x, y) d x \\
& \leq \frac{(b-m a) f(b, y)-(a-m b) f(a, y)}{2(b-a)} .
\end{aligned}
$$

Integrating (23) over $[a, b]$ and (24) over $[c, d]$ we get

$$
\begin{aligned}
& \frac{1}{b-a} \int_{a}^{b} \frac{f(x, m d)}{m} d x-\frac{d-c}{2(b-a)} \int_{a}^{b} \frac{\partial f(x, m d)}{\partial y} d x \\
& \quad \leq \frac{1}{(b-a)(d-c)} \int_{a}^{b} \int_{c}^{d} f(x, y) d y d x \\
& \quad \leq \frac{1}{b-a} \int_{a}^{b}\left(\frac{(d-m c) f(x, d)-(c-m d) f(x, c)}{2(d-c)}\right) d x
\end{aligned}
$$

and

$$
\begin{aligned}
& \frac{1}{d-c} \int_{c}^{d} \frac{f(m b, y)}{m} d y-\frac{b-a}{2(d-c)} \int_{c}^{d} \frac{\partial f(m b, y)}{\partial x} d y \\
& \leq \frac{1}{(b-a)(d-c)} \int_{a}^{b} \int_{c}^{d} f(x, y) d y d x \\
& \quad \leq \frac{1}{d-c} \int_{c}^{d}\left(\frac{(b-m a) f(b, y)-(a-m b) f(a, y)}{2(b-a)}\right) d y,
\end{aligned}
$$

respectively.

Adding (25) and (26) we get (22). 


\section{Competing interests}

The authors declare that they have no competing interests.

\section{Authors' contributions}

All authors contributed equally.

\section{Author details}

${ }^{1}$ COMSATS Institute of Information Technology, Kamra Road, Attock, 43600, Pakistan. ${ }^{2}$ Government Degree College, Peshawar, Pakistan.

\section{Acknowledgements}

The research of the first and third authors is partially funded by the COMSATS Institute of Information Technology, Islamabad, Pakistan.

Received: 17 July 2015 Accepted: 1 September 2015 Published online: 17 September 2015

\section{References}

1. Mitrinoviśc, DS, Pečarić, JE, Fink, AM: Classical and New Inequalities in Analysis. Kluwer Academic, Dordrecht (1993)

2. Niculescu, CP, Persson, LE: Convex Functions and Their Applications. A Contemporary Approach. Springer, New York (2006)

3. Pečarić, JE, Proschan, F, Tong, YL: Convex Functions, Partial Ordering, and Statistical Applications. Academic Press, San Diego (1992)

4. Persson, L-E, Ragusa, MA, Samko, N, Wall, P: Commutators of Hardy operators in vanishing Morrey spaces. In: AIP Conf. Proc vol. 1493, p. 859 (2012) doi:10.1063/1.4765588

5. Hermite, C: Sur deux limites d'une intégrale définie. Mathesis 3, 82 (1883)

6. Hadamard, J: Étude sur les propriètès des fonctions entières et en particulier d'une fonction considerèe par Riemann. J. Math. Pures Appl. 58, 171-215 (1893)

7. Abramovich, S, Farid, G, Pečarić, JE: More about Hermite-Hadamard inequalities, Cauchy's means, and superquadracity. J. Inequal. Appl. 2010, Article ID 102467 (2010)

8. Butt, SI, Pečarić, JE, Rehman, AU: Non-symmetric Stolarsky means. J. Math. Inequal. 7(2), 227-237 (2013)

9. Dragomir, SS, Pečarić, JE, Sáandor, J: A note on the Jensen-Hadamard inequality. L'Anal. Num. Theor. L'Approx. (Romania) 19, 21-28 (1990)

10. Dragomir, SS, Barbu, D, Buşe, C: A probabilistic argument for the convergence of some sequences associated to Hadamard's inequality. Stud. Univ. Babeş-Bolyai, Math. 38, $29-34$ (1993)

11. Dragomir, SS: On Hadamard's inequality for convex functions on the co-ordinates in a rectangle from the plane. Taiwan. J. Math. 4, 775-788 (2001)

12. Dragomir, SS: On Hadamard's inequality for convex functions. Math. Balk. 6, 215-222 (1992)

13. Dragomir, SS: Refinements of the Hermite-Hadamard inequality for convex functions. JIPAM. J. Inequal. Pure Appl. Math. 6(5), Article ID 140 (2005)

14. Dragomir, SS: Some integral inequalities for differentiable convex functions. Contributions, Macedonian Acad. of Sci. and Arts (Scopie) 16, 77-80 (1992)

15. Pečarić, JE, Dragomir, SS: A generalisation of Hadamard's inequality for isotonic linear functionals. Rad. Mat. 7 , 103-107 (1991)

16. Pečarić, JE, Dragomir, SS: On some integral inequalities for convex functions. Bull. Mat. Inst. Pol. lasi 36, 19-23 (1990)

17. Dragomir, SS: Two mappings in connection to Hadamard's inequalities. J. Math. Anal. Appl. 167, 49-56 (1992)

18. Toader, GH: Means and convexity, on generalization of convexity. Mathematica 30(53), 83-87 (1988)

19. Mitrinović, DS, Lačković, IB: Hermite and convexity. Aequationes Math. 28, 225-232 (1985)

20. Alomari, M, Darus, M: Co-ordinated s-convex function in the first sense with some Hadamard-type inequalities. Int. J. Contemp. Math. Sci. 3(32), 1557-1567 (2008)

21. Alomari, M, Darus, M: On the Hadamard's inequality for log-convex functions on the coordinates. J. Inequal. Appl. 2009, Article ID 283147 (2009)

22. Latif, MA, Alomari, M: Hadamard-type inequalities for product two convex functions on the co-ordinates. Int. Math. Forum 4(47), 2327-2338 (2009)

23. Ozdemir, ME, Set, E, Sarikaya, MZ: New some Hadamard's type inequalities for co-ordinated $m$-convex and $(\alpha, m)$-convex functions. Hacet. J. Math. Stat. 40(2), 219-229 (2011)

24. Toader, GH: Some generalizations of convexity. In: Proc. Colloq. Approx. Optim, Cluj Napoca (Romania), pp. 329-338 (1984)

25. Dragomir, SS, Toader, GH: Some inequalities for m-convex functions. Stud. Univ. Babeş-Bolyai, Math. 38(1), 21-28 (1993) 